\title{
(-) antex \\ Seizure-induced unilateral posterior dislocation of the shoulder: a diagnosis not to be missed
}

\author{
Andrew Roy McKean, ${ }^{1}$ Shankar Kumar, ${ }^{2,3}$ Greg Michael McKean, ${ }^{4}$ Demitrios Tzias ${ }^{5}$
}

\begin{abstract}
${ }^{1}$ Department of Plastic Surgery, Chelsea and Westminster Hospital NHS Foundation Trust, London, UK

${ }^{2}$ Centre for Medical Imaging, University College London, London, UK

${ }^{3}$ Imaging Department, University College London Hospitals NHS Foundation Trust, London, UK ${ }^{4}$ Department of Trauma and Orthopaedic Surgery, University Hospital Ayr, Ayr, UK ${ }^{5}$ Department of Radiology, Ashford and St Peter's Hospitals NHS Foundation Trust, Surrey, UK
\end{abstract}

\section{Correspondence to} Dr Andrew Roy McKean, andrew.mckean@nhs.net

Accepted 14 March 2018

\section{SUMMARY}

A man in his mid-50s with a history of bladder carcinoma presented to the Emergency Department (ED) following a witnessed tonic-clonic seizure. Computed Tomography (CT) scanning of his brain revealed an indeterminate mass lesion in the left parietal region. The patient described bilateral shoulder pain prompting plain film radiographs with axial views, but no obvious abnormality was identified by the ED staff. Staging CT scanning did not reveal any evidence of underlying malignancy but revealed dislocation of the left humeral head with a large reverse Hill-Sachs lesion and posteriorly displaced reverse Bankart lesion. Manipulation under anaesthesia and closed reduction of the left shoulder was undertaken successfully. This case report reminds the clinician to maintain a high index of suspicion for posterior dislocation of the shoulder following seizures and to perform appropriate imaging promptly.

\section{BACKGROUND}

Posterior dislocation of the shoulder is an uncommonly encountered injury accounting for 2\%-5\% of all shoulder dislocations. ${ }^{1}$ It has been estimated that anterior glenohumeral dislocations are nearly 22 times more common than posterior dislocations. ${ }^{2}$ The majority of posterior dislocations are associated with high-velocity trauma and epileptic seizures.

Despite it scarcity in clinical practice, it is important to be alert for posterior shoulder dislocation as delayed diagnosis and treatment can result in significant morbidity including chronic pain, stiffness and functional disability. ${ }^{3}$ Indeed, such injuries are often difficult to diagnose and overlooked with up to $80 \%$ of posterior dislocations being missed on initial clinical examination. ${ }^{1}$

The purpose of this case report is to remind the clinician to have heightened suspicion for posterior shoulder dislocation in patients who present following seizures and to undertake appropriate and timely radiological investigation.

\section{CASE PRESENTATION}

A 56-year-old man presented to hospital following a witnessed tonic-clonic seizure that self-terminated within 5 minutes. Prior to this event, he had been well with no intercurrent illness or preceding symptoms. There was no evidence of urinary incontinence or tongue biting, but he was noted to be in a postictal state with the paramedics.
He had a history of bladder carcinoma treated curatively without surgical intervention and was not a known epileptic, having not sustained a seizure previously. He seldom consumed alcohol and had no relevant family history.

On arrival to the Emergency Department (ED), his Glasgow Coma Score was 15 with no demonstrable focal neurology. Observations were all within normal limits and he had no features consistent with infection. He described bilateral shoulder pain that was slightly worse on the left side. On clinical examination of the shoulders, globally reduced range of movement on the left side was noted.

\section{INVESTIGATIONS}

Left shoulder anteroposterior (AP) and lateral radiographs were obtained (figure 1 ) but the abnormality was not initially detected in the ED. A non-contrast computed tomography (CT) brain scan indicated a possible mass lesion with surrounding oedema in the left parietal region (figure 2).

The next day, further evaluation of the brain was undertaken with magnetic resonance imaging (MRI)of the head with gadolinium, and a CT scan of the chest, abdomen and pelvis was performed for staging purposes. The MRI scan confirmed the presence of a solitary enhancing mass lesion in the left posterior temporal lobe with oedema in the adjacent white matter (figure 3). CT chest, abdomen and pelvis imaging did not reveal any evidence of malignancy but confirmed dislocation of the left humeral head with a reverse Hill-Sachs lesion and a reverse Bankart displaced glenoid fracture (figure 4).

\section{TREATMENT}

Initially, the patient was resuscitated with intravenous fluids in the ED. Following medical review, he was admitted and commenced on a short course of dexamethasone and levetiracetam as per the local neurology team.

In view of the CT scan findings, urgent orthopaedic review was obtained and the patient subsequently underwent manipulation under anaesthesia and closed reduction of the left shoulder. Referral to the regional neuro-oncology multidisciplinary team (MDT) meeting was also made for further evaluation and input with regards to the nature of the left parietal mass lesion.
Kumar S, McKean GM, et al.

BMJ Case Rep Published

Online First: [please

include Day Month Year]

doi:10.1136/bcr-2017-

223160 


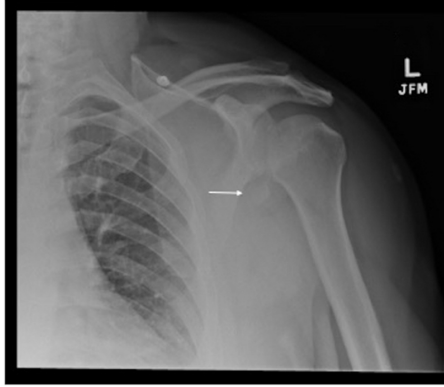

A

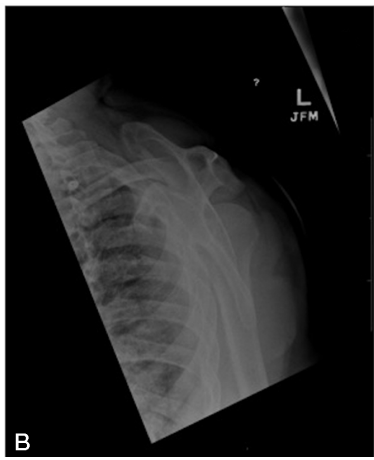

Figure 1 Plain radiographs of the left shoulder. (A) Anteroposteriorflattening and irregularity of the medial humeral head with a displaced fracture fragment originating from the inferior glenoid (arrow). (B) Lateral ( $Y$ view) showing the humeral head displaced posteriorly.

\section{OUTCOME AND FOLLOW-UP}

The patient underwent successful closed reduction of the left shoulder dislocation and is to be followed up at the local orthopaedic upper limb clinic in due course. Following discussion at the regional neuro-oncology MDT, the intracranial lesion was of indeterminate nature and close follow-up with repeat MRI scanning of the brain in 4-6 weeks was advised. The patient made an excellent recovery and was discharged on levetiracetam therapy with referral to the local 'First Fit' clinic.

The follow-up MRI brain scan with gadolinium showed stable appearances, not consistent with an underlying benign or malignant tumour, correlating clinically with no further seizures and a full recovery. However, diagnostic uncertainty remains and alternate diagnoses such as an insidious infective process will be considered in due course.

\section{DISCUSSION}

Posterior dislocation of the shoulder, whether unilateral or bilateral, is nearly always pathognomonic of a seizure in an atraumatic setting. ${ }^{4}$ During posterior shoulder dislocation, the humeral head impacts on the posterior aspect of the glenoid and due to rotational movements, grinding can occur resulting in cortication and a secondary cartilaginous injury. Therefore, timely diagnosis and definitive treatment are imperative to avoid resulting morbidity.

To the best of our knowledge, the exact sequence of events outlined in this case report of an indeterminate intracranial mass lesion causing a witnessed tonic-clonic seizure, leading to unilateral posterior dislocation of the shoulder, has not been described
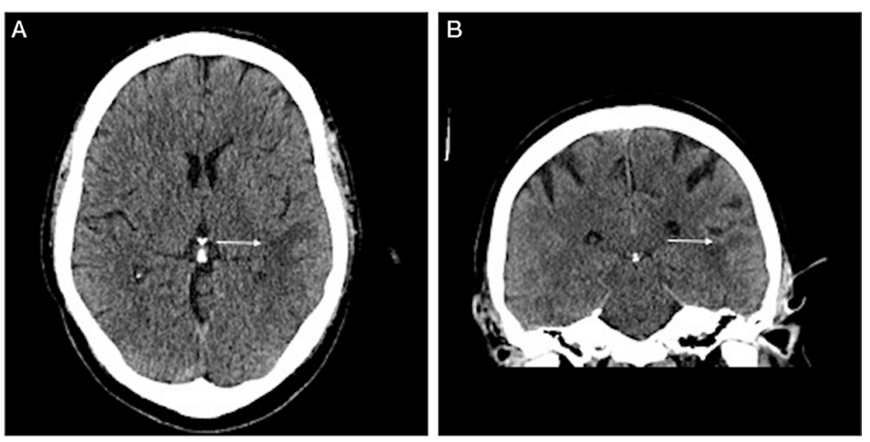

Figure 2 Non-contrast CT brain demonstrating an asymmetrical low density lesion in the left temporal lobe, in keeping with oedema on axial (A) and coronal slices (B), arrowed.
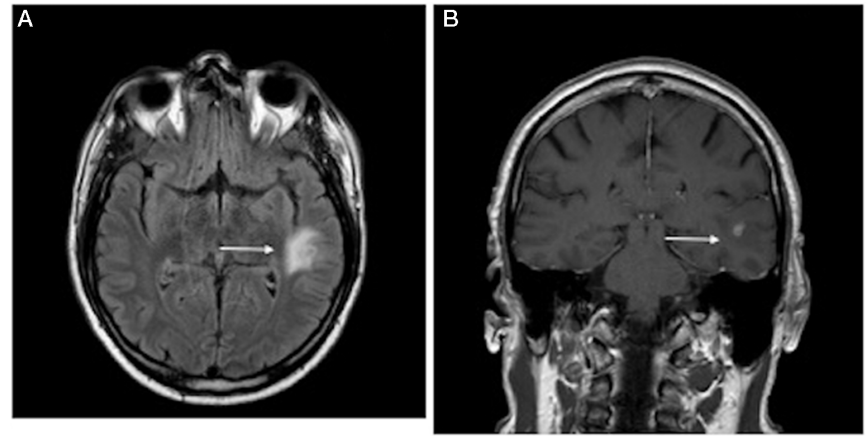

Figure 3 MRI with gadolinium of brain. Axial FLAIR (A) demonstrating an area of high signal in keeping with oedema of the left temporal lobe. Coronal T1 (B) with gadolinium administration demonstrating enhancing lesion central to the area of oedema, arrowed. FLAIR, fluidattenuated inversion recovery.

previously in the literature. In 1990, Elliott described a case of a patient who woke up with pain in the right shoulder and was subsequently found to have a two-part posterior fracture dislocation of the right shoulder. ${ }^{5}$ In recognition of the fact that in the absence of trauma, these injuries usually occur secondary to a seizure, the patient underwent a CT head scan which revealed a space occupying lesion in the left frontal lobe. Later excisional biopsy confirmed a diagnosis of benign macrocystic meningioma.

Kilicoglu et al reported a patient presenting with headache and bilateral shoulder pain, who was found to have bilateral posterior shoulder dislocation with impaction fractures of both humeral heads. ${ }^{6}$ Again, although this patient had no history of seizures, he underwent cranial MRI imaging which revealed a mass lesion in the right temporal lobe involving the uncus. Intralesional excision of the tumour and subsequent histological analysis confirmed Grade II atsrocytoma.

These cases highlight the need to consider a seizure-related aetiology for posterior shoulder dislocation in the absence of trauma. Diagnostic delay of posterior shoulder dislocation is common and can be due to late presentation, non-compliance with clinical examination and imaging, and inadequate or suboptimal radiological investigation.

Acute posterior dislocation of the shoulder is characteristically painful. However, ability to illicit pain on palpation of the affected shoulder joint may be reduced in the postictal state or

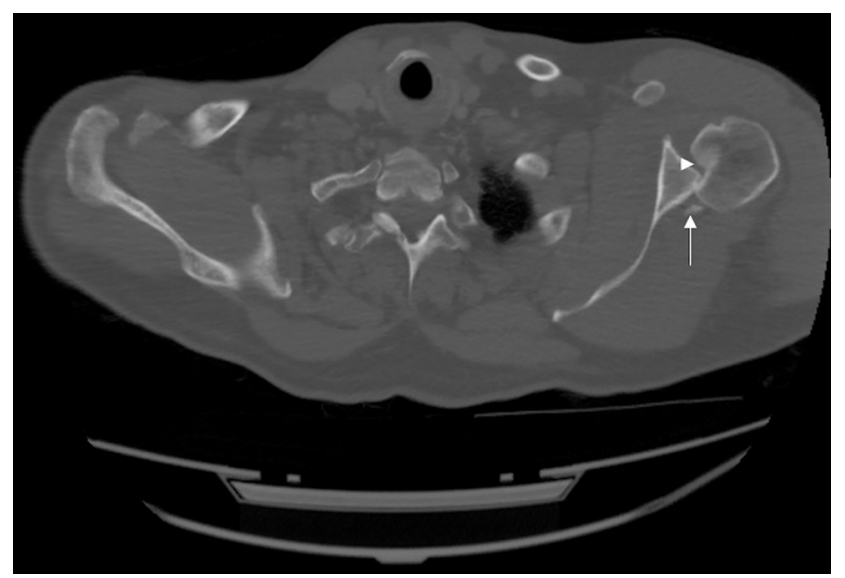

Figure 4 Axial CT chest. Left humeral head posteriorly subluxed with reverse Hill-Sachs defect (arrowhead) and posteriorly displaced reverse Bankart lesion (arrow). 
in the presence of acute intoxication. The affected shoulder is likely to be held in internal rotation. ${ }^{7}$ There will be a mechanical block to external rotation caused by engagement of the anterior humeral head defect on the posterior aspect of the glenoid. Rotator cuff function and neurovascular status should also be assessed to exclude any concurrent rotator cuff or neurovascular injury.

In the present case, despite anteroposterior (AP) and lateral radiographs of the shoulder, posterior dislocation of the left shoulder was only identified serendipitously from the staging CT scan. This illustrates the necessity for specific axillary views (apical oblique, Velpeau or modified axial) especially when the local protocol consists of only AP and lateral views. These radiographs are often suboptimal as the patient may be unable to abduct the arm. Obtaining an axillary view has the added practical benefit that it can be obtained with the arm in a sling, without the need for abduction. Additionally, classically described radiographic signs of posterior dislocation on AP radiographs are unreliable. ${ }^{7}$ Notably, a centre that adopted the protocol of obtaining an AP and axillary view at the initial assessment, reported a delayed diagnosis in only $9 \%$ of patients who presented acutely. ${ }^{2}$ Additional views, including the Bernageau (glenoid profile) view, are effective in demonstrating the degree of bone loss of the glenoid or humeral head. ${ }^{8}$ Indeed, obtaining these views bilaterally can be useful to highlight potential underlying abnormalities such as posterior dislocation. CT is also useful to confirm the diagnosis and is essential prior to any attempt at closed reduction. ${ }^{3}$ Non-contrast MRI is effective in diagnosing soft tissue pathologies including labral, rotator cuff and glenohumeral ligament injuries. ${ }^{9}$ MR arthrography is a reliable alternative but its use is limited by its invasive nature and relative lack of availability compared with CT. ${ }^{9}{ }^{10}$ Historically, non-contrast CT has been used to assess bony Bankart and Hill-Sachs lesions, but more recently, MRI has been shown to be equivalent to CT to assess bone loss of the glenoid and humeral head. ${ }^{10} \mathrm{CT}$ is unable to assess labroligamentous or rotator cuff injuries which has implications if MRI is not performed preoperatively. Understanding the severity and extent of soft-tissue injury may influence the surgeon's decision-making process on optimal surgical management.

There is no evidence-based consensus on the optimal surgical management for such injuries. ${ }^{7}$ Despite this, disimpaction and bone grafting for small defects $(<25 \%$ of thehumeral head surface) within 2 weeks of the initial injury is advised. Small defects presenting 2 weeks after initial injury can be treated with subscapularis transfer. Larger defects $(40 \%-50 \%$ of the humeral head surface) should be filled with a structural allograft regardless of the time from injury to provide stability. Shoulder stability needs to be closely monitored postoperatively and residual instability may be due to capsulolabral disruption which may necessitate further repair.

\section{Learning points}

- Posterior shoulder dislocation is pathognomonic of seizures in the atraumatic setting.

- Diagnosis is often delayed or missed, leading to significant morbidity including chronic pain, stiffness and functional disability.

- Appropriate imaging with axillary views in addition to traditional axial radiographs is essential for prompt identification of these injuries.

Contributors ARM and DT identified the case. ARM and GMM provided surgical input, DT and SK provided radiological input. ARM and SK drafted the initial version which was added to by GMM and DT. All authors approved the final version for submission.

Funding The authors have not declared a specific grant for this research from any funding agency in the public, commercial or not-for-profit sectors.

Competing interests None declared.

Patient consent Obtained.

Provenance and peer review Not commissioned; externally peer reviewed.

Open Access This is an Open Access article distributed in accordance with the Creative Commons Attribution Non Commercial (CC BY-NC 4.0) license, which permits others to distribute, remix, adapt, build upon this work non-commercially, and license their derivative works on different terms, provided the original work is properly cited and the use is non-commercial. See: http://creativecommons.org/ licenses/by-nc/4.0/

(C) BMJ Publishing Group Ltd (unless otherwise stated in the text of the article) 2018. All rights reserved. No commercial use is permitted unless otherwise expressly granted.

\section{REFERENCES}

1 Rouleau DM, Hebert-Davies J. Incidence of associated injury in posterior shoulder dislocation: systematic review of the literature. J Orthop Trauma 2012;26:246-51.

2 Robinson CM, Seah M, Akhtar MA. The epidemiology, risk of recurrence, and functional outcome after an acute traumatic posterior dislocation of the shoulder. $J$ Bone Joint Surg Am 2011;93:1605-13.

3 Kokkalis ZT, lliopoulos ID, Antoniou G, et al. Posterior shoulder fracture-dislocation: an update with treatment algorithm. Eur J Orthop Surg Traumatol 2017;27:285-94.

4 Brown RJ. Bilateral dislocation of the shoulders. Injury 1984;15:267-73.

5 Elliott DS. Brain tumour presenting as a dislocated shoulder. J Bone Joint Surg Br 1990;72:925.

6 Kilicoglu O, Demirhan M, Yavuzer Y, et al. Bilateral posterior fracture-dislocation of the shoulder revealing unsuspected brain tumor: case presentation. J Shoulder Elbow Surg 2001;10:95-6.

7 Robinson CM, Aderinto J. Posterior shoulder dislocations and fracture-dislocations. J Bone Joint Surg Am 2005;87:639-50.

8 Murachovsky J, Bueno RS, Nascimento LG, et al. Calculating anterior glenoid bone loss using the Bernageau profile view. Skeletal Radiol 2012;41:1231-7.

9 Pavic R, Margetic P, Bensic M, et al. Diagnostic value of US, MR and MR arthrography in shoulder instability. Injury 2013;44(Suppl 3):S26-S32.

$10 \mathrm{Oh} \mathrm{JH}$, Kim JY, Choi JA, et al. Effectiveness of multidetector computed tomography arthrography for the diagnosis of shoulder pathology: comparison with magnetic resonance imaging with arthroscopic correlation. J Shoulder Elbow Surg 2010;19:14-20. 
Copyright 2018 BMJ Publishing Group. All rights reserved. For permission to reuse any of this content visit http://group.bmj.com/group/rights-licensing/permissions.

BMJ Case Report Fellows may re-use this article for personal use and teaching without any further permission.

Become a Fellow of BMJ Case Reports today and you can:

- Submit as many cases as you like

- Enjoy fast sympathetic peer review and rapid publication of accepted articles

- Access all the published articles

- Re-use any of the published material for personal use and teaching without further permission

For information on Institutional Fellowships contact consortiasales@bmjgroup.com

Visit casereports.bmj.com for more articles like this and to become a Fellow 